\title{
Marcel Proust 4. Proust au tournant des siècles I (textes réunis par Bernard Brun et Juliette Bassine
}

\section{Geneviève Henrot Sostero}

\section{(2) OpenEdition}

1 Journals

\section{Edizione digitale}

URL: http://journals.openedition.org/studifrancesi/36658

DOI: 10.4000/studifrancesi.36658

ISSN: 2421-5856

\section{Editore}

Rosenberg \& Sellier

\section{Edizione cartacea}

Data di pubblicazione: 1 juillet 2005

Paginazione: 198-199

ISSN: 0039-2944

\section{Notizia bibliografica digitale}

Geneviève Henrot Sostero, «Marcel Proust 4. Proust au tournant des siècles I (textes réunis par Bernard Brun et Juliette Bassine», Studi Francesi [Online], 145 (XLIX | I) | 2005, online dal 30 novembre 2015, consultato il 19 avril 2021. URL: http://journals.openedition.org/studifrancesi/36658 ; DOI: https:// doi.org/10.4000/studifrancesi.36658

Questo documento è stato generato automaticamente il 19 avril 2021.

\section{(c)}

Studi Francesi è distribuita con Licenza Creative Commons Attribuzione - Non commerciale - Non opere derivate 4.0 Internazionale. 


\title{
Marcel Proust 4. Proust au tournant des siècles I (textes réunis par Bernard Brun et Juliette Bassine
}

\author{
Geneviève Henrot Sostero
}

\section{NOTIZIA}

Marcel Proust 4. Proust au tournant des siècles I (textes réunis par Bernard Brun et Juliette Bassine), Paris-Caen, Minard, Lettres Modernes, 2004, pp. 292.

1 In occasione del Congresso internazionale organizzato da Juliette Hassine e Judith Kauffmann all'Università di Bar-Ilan (Ramat-Gan) in Israele, dal 26 al 28 novembre 2001, vengono pubblicati nel presente volume diciassette interventi, senza distinzione tra quelli che furono realmente pronunciati in occasione del congresso, quelli che, pur scritti per quell'occasione, non furono presentati al congresso, e quelli che avevano un'altra destinazione originaria. Il motivo? Rendere conto in modo unitario delle direzioni più recenti della critica prustiana, con un primo volume che avrà prossimamente un seguito. La critica genetica, votata all'analisi dei manoscritti, vi regna incontrastata in una corposa prima sezione. Vengono sondate le archeologie rispettive del barone di Charlus, dell'articolo pubblicato dal narratore nel Figaro, della rappresentazione degli Ebrei nella società. È proprio sicuro che Proust, che tratta il versante femminile dell'omosessualità già nei suoi primissimi racconti (1895-99), aspetti gli anni 1908 per affrontare il suo versante maschile? È proprio attendibile l'asimmetria genetica di trattamento tra i due versanti dell'omosessualità (Sodoma e Gomorra)? Significherebbe dimenticare la precoce nascita di un personaggio fondamentale nella pittura dell'omosessualità maschile (il barone di Charlus), nelle vesti del visconte di Lomperolles: Nathalie MAURIAC DYER dà, oltre al commento, la trascrizione integrale (4 pagine) della narrazione manoscritta (Jean Santeuil, $\left.\mathrm{f}^{\circ} 460 \mathrm{r}^{\circ}\right)(M$ de Lamperolles dans Jean Santeuil ou un aspect négligé de la genèse de Charlus, pp. 9-21). Mireille NATUREL, in Le Fabuleux destin de l'article dans 'Le Figaro' (pp. 23-39), ricostituisce e 
ripercorre la sinuosa genesi della scena in cui l'eroe scopre un suo articolo nel quotidiano Le Figaro, illustrando come la materia del romanzo si nutra dei primi esperimenti di scrittura giornalistica. Bernard BRUN, Sur quelques plaisanteries antisémites dans les manuscrits de rédaction de Proust pp. 41-52, rintraccia la genesi delle principali battute antisemitiche deI romanzo, cercando di capire come, quando, perché e con quali voci narrative il tema si ampli dopo la prima guerra mondiale. Tirando le somme delle prime due ondate genetiche nel campo della critica francese (quella dei lavori fondatori degli anni 1970, e quella delle edizioni degli anni 1980-1990), Jean-Marc QUARANTA interroga nuovamente il ruolo della critica genetica e il modo in cui essa abbia modificato profondamente lo sguardo sulla letteratura; il punto di vista epistemologico, nel senso "archeologico" che gli conferisce Foucault, illumina costanti e Torture discorsive attorno alla fondamentale "critica dell'intelligenza" ripetutamente argomentata da Proust, che mira, secondo il critico, a liberare l'arte dalla mediazione della filosofia (Génétique et intertextualité: pour une lecture épistémologique du corpus proustien, pp. 53-74). Françoise LERICHE, in La théorie estétique proustienne à l'épreuve de la génétique (pp. 75-108), considera le brutte copie come tracce di un processo mentale in cui l'espressione agisce a ritroso sul pensiero e lo confronta con le proprie aporie: il progetto (o intenzione teorica) si discosta a volte dallo "scenario" di tale idea nella finzione.

2 L'approccio intertestuale, al quale viene dedicata la seconda sezione del volume, riflette sul problema della lettura e sui rapporti tra lettura e scrittura. Thierry ALCOLOUMBRE riscopre in Aristote utili concetti alla lettura di Proust (Aristote lecteur de Proust pp. 111-13). Si popola di anime affini il contesto letterario del Nostro: Stéphane CHAUDIER scorge in Boylesve una copia in miniatura del grande Proust, a causa di un'analoga sensibilità nei confronti del patrimonio dell'infanzia (Proust et Boylesve: récits d'enfance entre deux siècles pp. 133-144); Anne SIMON decifra sotto molti brani prustiani un palinsesto in cui traspaiono temi, motivi e termini prediletti dello studioso della natura e delle api, Maurice Maeterlinck (Proust lecteur de Maeterlinck pp. 145-160); Jo YosHIDA segue il filo dolente e sanguigno di san Sebastiano nelle similitudini e metafore che dipingono il personaggio di Legrandin (Le 'Martyr de saint Sébastien' et Marcel Proust pp. 161-174). Edward BIZUB, oltre a sopravvalutare la fortuna dell"'epifania" joyciana nella critica proustiana (solo J.-M. Quaranta usa il concetto in modo non aneddotico), non si accorge che il commento Proust/Joyce si morde la coda, giacché torna semplicemente a Joyce ciò che gli era stato scientemente preso in prestito: finta somiglianza dunque, basata sull'uso di un termine del resto poco legittimo nel caso di Proust (Proust et Joyce: une rencontre autour de l'épiphanie pp. 175-186). Infine, Juliette Hassine si chiede quanto la poetica dello stile di Virginia Woolf debba a quel la di Proust (Virginia Woolf et Marcel Proust: "remarques sur le style" pp. 187-197).

La terza e ultima sezione del volume prende titolo dal primo studio, di Annick BOUILLAGUET (L'écriture infinie, pp. 201-210). La critica tradizionale, nel proclamare il valore catartico dei "pastiche" per Proust, misconosce due verità: la prima, che Proust pratica il "pastiche" in tanti brani mimetizzati nel romanzo, in cui tale funzione non si manifesta di certo come Dei Pastiches de L'Affaire Lemoine la seconda, che non bisogna fidarsi troppo delle dichiarazioni di Proust al riguardo. Bellissimo saggio di traduttologia, la riflessione di Helit YESHURUN sulla sua esperienza di traduttrice della Recherche in ebraico: tradurre Proust, con tutta la polpa delle sue sensazioni concrete e sussurrate, significa foggiare di sana pianta una espressività nuova a quella lingua 
spigolosa, quadrata e asciutta che gli Ebrei hanno cristallizzato nel suo uso sacro (A la recherche de l'hébreu, pp. 211-222). Vera LASRY sottolinea nella descrizione dei corpi fittizi una costante tensione fra trasparenza e opacità, che conferisce alla loro descrizione il vero slancio dinamico (Regards sur le corps humain dans l'écriture proustienne, pp. 223-233). Elisheva ROSEN soppesa delicatamente il modo innovativo in cui Proust filtra i rapporti tra la sua opera e i realia sociali del suo tempo (Mondanités proustiennes: le tournant de Sodome et Gomorrhe, pp. 235-248). Jean DELACOUR convoglia un recentissimo sapere neurologico e cognitivo nella lettura della memoria involontaria (Les Réminiscences proustiennes et l'étude scetifique de la mémoire, pp. 249-271), mentre Geneviève HENROT, prendendo lo spunto dalla definizione saussuriana del segno linguistico, analizza i rapporti tra teoria del segno e ricordo involontario, dal segno della memoria alla memoria del segno (L'Architexture du signe proustien, pp. 273-291). 